\title{
Using Genetic Algorithm to Estimate (RNA) Estimator
}

*Ban Ahmed Mitras

**Farah Saad Nashat

*dr.banah.mitras@gmail.com
College of Computer Sciences and Mathematics
University of Mosul
**College of Education. University of Dohuk

\section{Received on: 16/08/2010}

Accepted on: 10/11/2010

\begin{abstract}
In this paper the genetic algorithm has been used to estimate the parameter $\theta$ which exist in Boltzmann Distribution which controls the structure of the Ribo Nucleic Acid (RNA). Two algorithms have been suggested. The first found the value of the estimator which maximizes the likelihood function of Boltzmann Distribution. The second minimized the generation constraint of Boltzmann Distribution by using the genetic algorithm. Matlab (7.0) has been used in writing the programs of algorithms and achieved the following results: The maximum value for the likelihood estimator for Boltzmann Distribution appear at the value -4.1614 where the value of $\theta$ is 0.1457 , and the minimum value for the Constraint Generation for Boltzmann Distribution appear at the value $0.951039101 * 1^{7}$ where the value of $\theta$ is -4.4066 .
\end{abstract}

Keyword: Genetic Algorithm, Boltzmann Distribution, RNA Estimator.

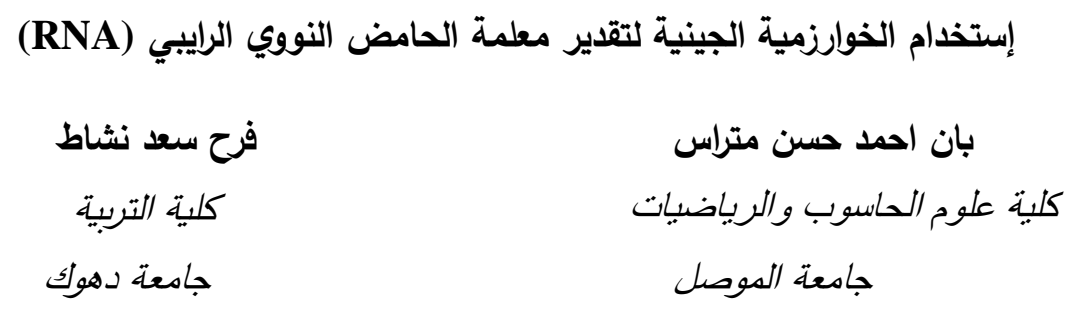

تاريخ قبول البحث:10/11/2010

تاريخ استلام البحث:16/08/2010

\footnotetext{
الملخص

تم في هذا البحث استخدام الخوارزمية الجينية لتقدير المعلمة $\theta$ الموجودة في توزيع بولتزمان الذي يخضع

لـه تركيب الحامض النووي الرايبي (RNA) إذ تم اقتراح خوارزميتان أولهما تقوم بإيجاد قيمة المقدر الذي يعظم دالة الترجيح لتوزيع بولتزمان، والثانية تقوم بتقليل دالة قيد الجيل لتوزيع بولتزمان باستخدام الخوارزمية الجينية، ولقد استخدم نظام Matlab (7.0 في كتابة برامج الخوارزميات والتي حصلنا من خلالها على النتائج التالية: أعظم قيمة لمقدر الترجيح لتوزيع بولتزمان ظهرت عند القيمة 94.1614- حيث كانت قيمة $\theta$ هي

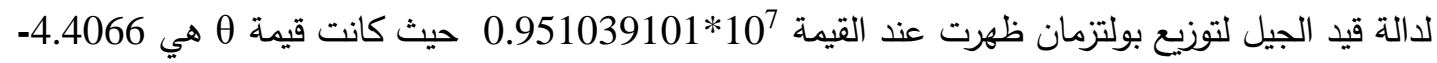

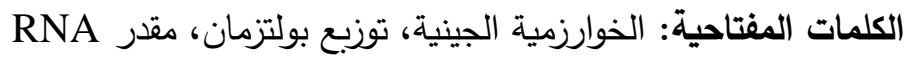


1. مقدمة: Introduction

شهد القرن العشرين تقدماً هائلا في الأساليب العلمية المستخدمة في البحث العلمي في حقول المعرفة كافة ويشمل هذا التطور علم الإحصاء الذي أصبح علماً مستقلاً له أهميته بوصفه وسيلة للبحث العلمي وأداته، ونظراً لأهميته واستخدامه في العديد من مجالات الحياة، فقد اهتم العديد من الباحثين به وقاموا بدراسته وادخاله في الإني بعض المجالات الحياتية ومنها دراسة بعض الأحماض النووية التي تدخل في التركيب الداخلي لجسم الإنسان ومن لهن

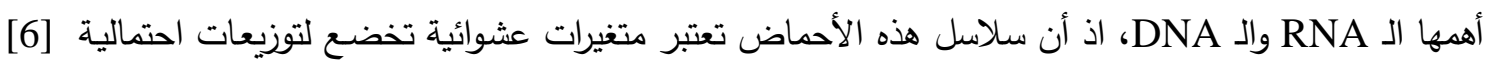

والمقصود بتوزيع المتغير العشوائي (أو التوزيع الإحتمالي لمتغير عشوائي) بأنه تعريف السلوك الرياضي

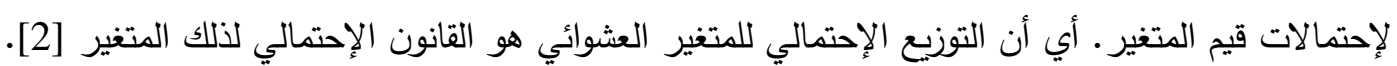

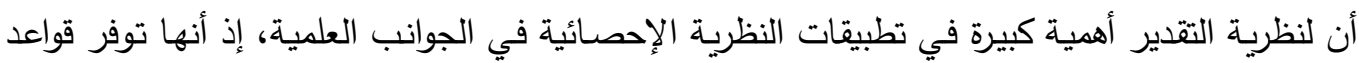
يتم بموجبها تقدير معلمات مجهولة لظاهرة معينة في الواقع العملي ولمختلف المجالات.

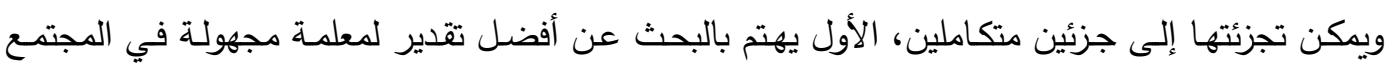
ويسمى بالتقدير النقطي (Point Estimation)، ويهتم الجزء الثاني بالبحث عن أفضل فئن فترة يمكن حصر قيمة

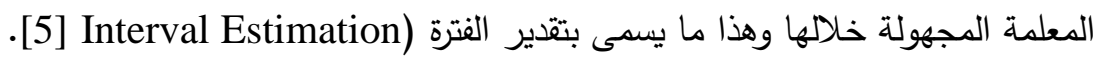

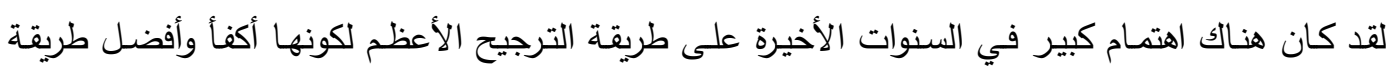

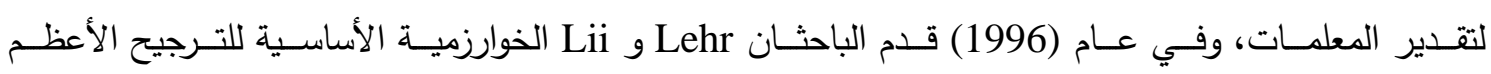
التي تعطي تقديرات ثابتة وكفووة للمعلمات، إن تقدير العينات الكبيرة (Maximum Likelihood Estimation) يمكن أن تكون مكلفة أو حتى مستحيلة ولهذا طور كل من Xu و Vogl في عام (2000) طريقة الترجيح الأعظم وتمكنا من تقدير معلمات هذه العينات. وهناك عدة بحوث تختص بتقدير المعلمات باستخدام توزيع بولتزمان الذي يمثل تركيب سلسلة الـ RNA وفيما

$$
\text { يلي بعض من هذه الأعمال و البحوث: }
$$

وصف Pond وآخرون في عام (2006) طريقة أساسها دالة الإمكان لإختيار النموذج التطوري باستخدام

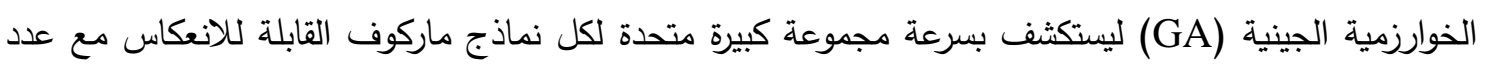
ثابت لإحلال النسب. وحققوا أيضاً فائدة لعدة مقاييس مسافة للمقارنة والتغاير للنماذج التطورية المستنتجة [10]. وفي العام نفسه وصف Ding وآخرون إجراء بديل لتمثيل تركيب الحامض الرايبي المراسل mRNA، لطعايل

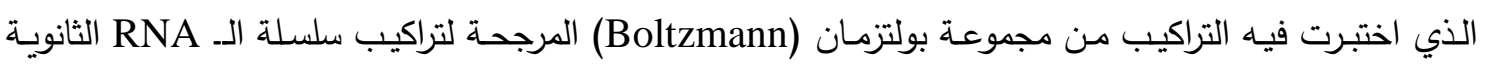
المتجمعة، بالاعتمـاد على عينة عشوائية من طول طبيعي للإنسان وأخذ الـ mRNA منـه، واظهروا خصـائص

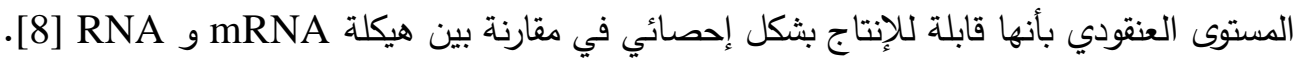

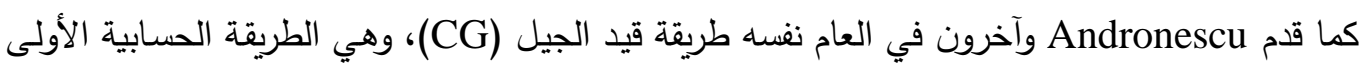
لتقدير معلمة الطاقة الحرة لسلسلة الـ RNA التي يجب أن تدرب بشكل كفوي على هيكلية المجموعات الكبيرة فضلاً عن بيانات الديناميكية الحرارية. وبينوا أن طريقة قيد الجيل (Constraint Generation) توظف مخططاً

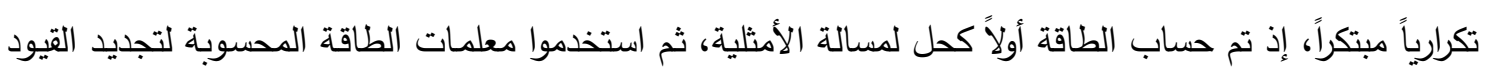

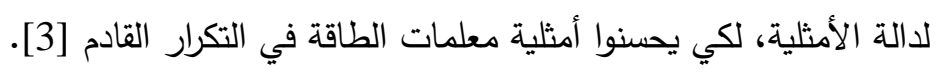


وقد استعمل الباحثان Chan و Ding في عام (2008) أسلوب العينات وجمعوا عناقيد للجموعة بولتزمان

لتركيب RNA (Boltzmann) تعديلاتهم العشوائية، وقاموا بتحليل أساس تركيب الـ MicroRNA المتمثل بـ (miRNA) لـ9 مجموعات مديزة

في هذا البحث تم تقدير معلمة توزيع بولتزمان المتمثل بمعلمة RNA باستذام الخوارزمية الجينية وذلك بـ

1 - ت تعظيم دالة الترجيح لتوزيع بولتزمان باستخدام الخوارزمية الجينية.

2. تقليل قيد الجيل لتوزيع بولتزمان باستخدام الخوارزمية الجينية.

Genetic Algorithm الخوارزمية الجينية:2

تتضمن الخوارزمية الجينية عدداً من الخطوات الأساسية، هذه الخطوات ثابتة ليختلف الدسائل ولكل

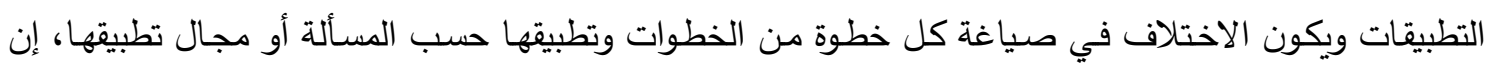
خطوات هذه الخوارزمية تكون مترابطة بعضها مع البعض الآخر ، ولا يمكن تطبيق هذه الخوارزمية على أية مسألة

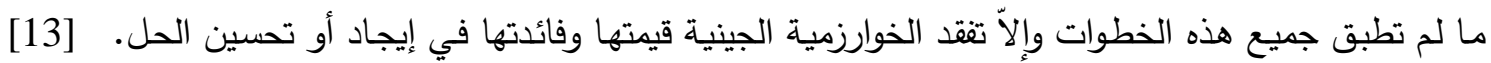
[12] [12] والثكل(1) يوضح المخطط العام للخوارزمية الجينية:

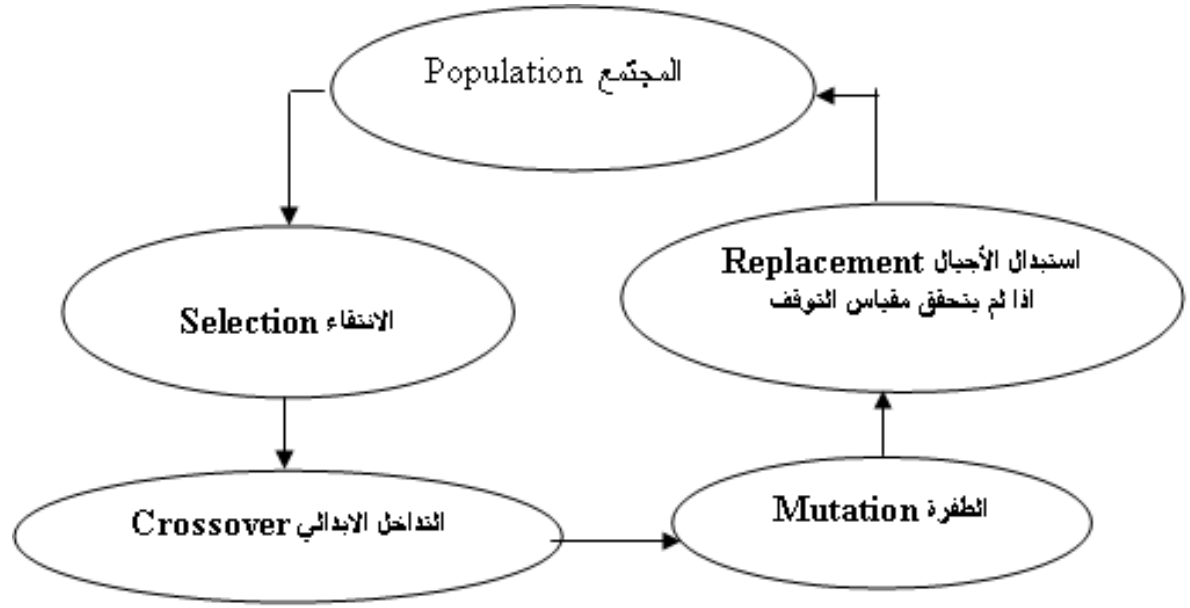

الثكل (1) يمثل خطوات الخوارزمية الجينية

خطوات عمل الخوارزمية الجينية: Working Steps in Genetic Algorithm

1-البداية Start: توليد مجتمع عشوائي من الكروموسومات، أي بمعنى إيجاد حلول مناسبة للمسألة. 2-Fitness هي تحويل دالة الهدف (Objective Function) إلى دالياقد دالة مناسبة للحل في الخوارزمية

3-مجتمع جديد New Population: توليد جيل جديد بتكرار الخطوات الآتية إلى أن يكتمل الجيل، وتتضمن: 
الاختيار Selection: يتم اختيار اثثين من الكروموسومات والدين (Parents Chromosomes) من

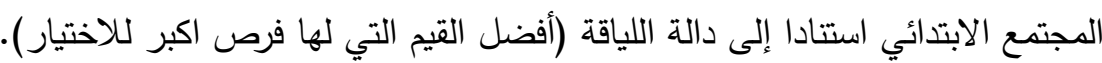

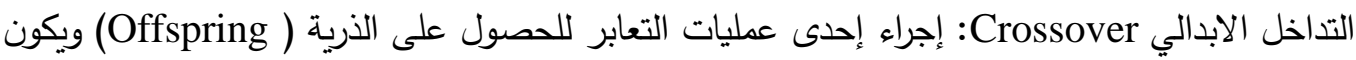
بين كروموسومين.

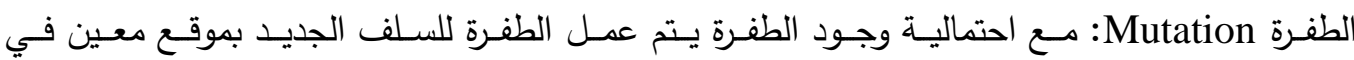
الكروموسوم، وتجري بين الجينات في الكروموسوم الواحد.

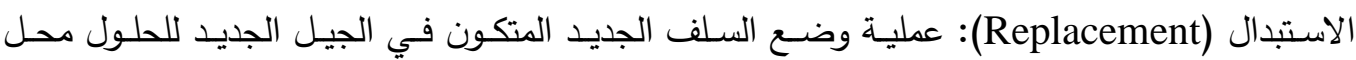
المجتمع الابتدائي. الاختبار Test: عند تحقق شرط التوقف، فإن الخوارزمية الجينية تتوقف وتعيد الحل الجيد من آخر جيل متكون. الدورة Loop: Lيتم الرجوع إلى الخطوة 2. سإن كل تكرار لهذه العملية يسمى بالجيل ( Generation)، وبعد نهايـة التتفيذ يقوم الباحث بتقديم تقرير عن لتصن الحقائق التي تم التوصل إليها.

\section{3. الأحماض النووية في الخلية: Nucleic of the Cell}

توجد الأحماض النووية في الخلية"، إذ أن الخلية هي وحدة البناء والوظيفة في الكائن الحي وهي على نوعين:الحامض النووي الرايبي (Ribo Nucleic Acid) ويرمز لـه اختصـاراً RNA مالحامض النووي الرايبي منقوص الأوكسجين (Deoxyribo Nucleic Acid) ويرمز له DNA [11]. إن موضوع دراستتا يتتاول الحامض النووي الرايبوسومي (RNA) وهو يوجد في كل من النواة والسايتوبلازم، حيث يوجد في النوية وفي الرايبوسومات وفي تراكيب أخرى. ويمكن تعريف (السايتوبلازم) بأنه جزء المادة الحية للخلية الذي يقع خارج النواة. وتعرف (النواة) على لعى أنها أكبر عضية متميزة داخل الخلية وللنواة أهمية كبيرة في نقل الصفات الوراثية وفي النشاط الأيضي للخلية [9].

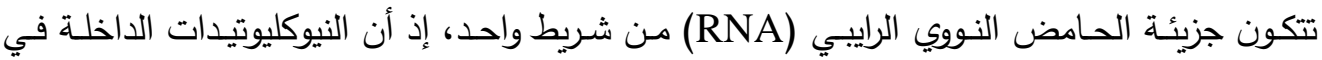
تركيب الـ RNA تتألف من ثلاث جزيئات بسيطة مرتبطة بعضها ببعض مباشرة وهي:

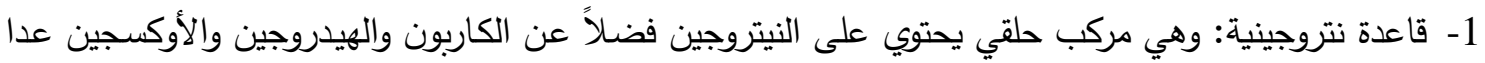
الأدنين الذي لا يحتوي بدوره على الأوكسجين، ويوجد نوعان منها: أ-البريميدينات (Pyrimidines): وتتكون من حلقة واحدة وتشمل القواعد الاتية:

1-السايتوسين (Cytosine) ويرمز لها (C).

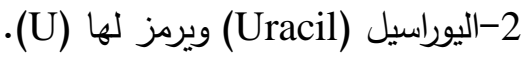

ب- البيورينات (Purines): وتتكون من حلقتين وتثمل:

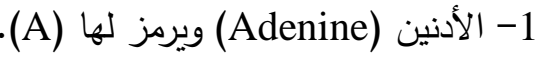

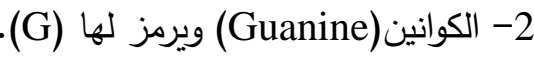

فالأدنين يرتبط دائماً مع اليوراسيل بآصرتين هيدروجينيتين A=U والسايتوسين يرتبط دائماً مع الكوانين

$$
\text { بثلاث أواصر هيدروجينية C }
$$


2- سكر خماسي الكاربون وهو الرايبوز وصيغته الجزيئية هي (C) 3-حامض الفنفوريك.

والثكل الآتي يبين العرض الهيكلي لسلسلة الـ RNA

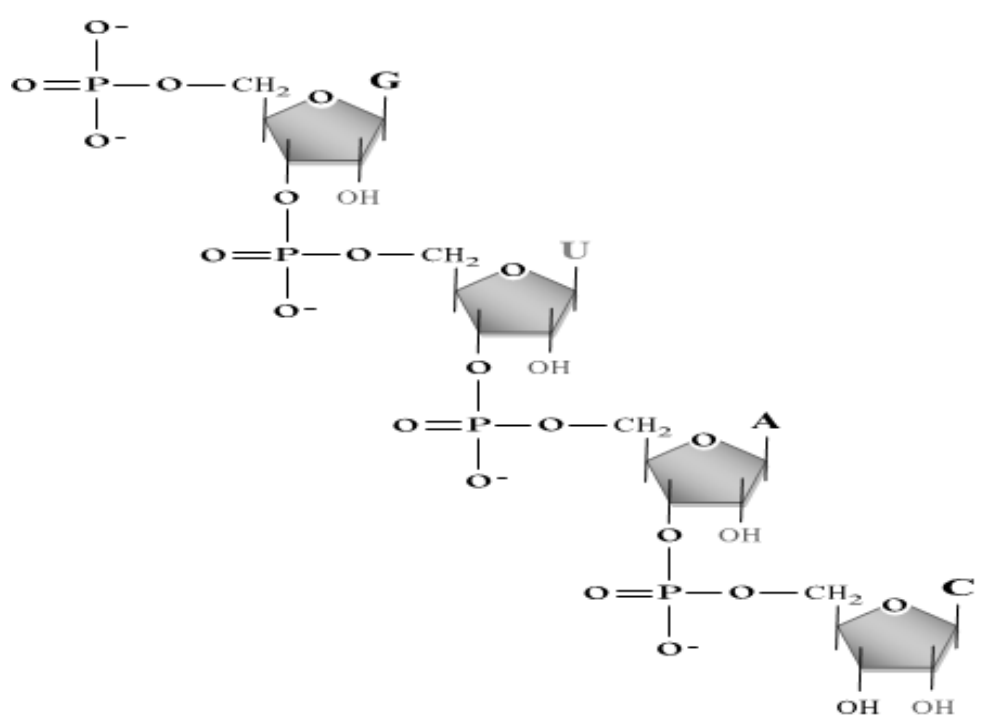

\section{الثكل ( 2 ) يمثل العرض الهيكلي لسلسلة الـ RNA}

وفي حالات نـادرة قد يكون الحامض النووي الراييوسومي RNA هو المـادة الوراثية كمـا في بعض الرواشح (الفيروسات)، ولكن أهميته أعظم وأعم في الأحياء بسبب الدور الذي يقوم به في عملية بناء البروتين بما في ذلك الأنزيمات.

ويمكن تمييز ثلاثة أنواع من الـ RNA وكلها تصنع في النواة وتتتقل إلى السايتوبلازم لتشارك في صنع البروتين بشكل خاص وهي:

1- الحامض الرايبي المراسل Messenger RNA (mRNA) ويقوم بنقل المعلومات الوراثية من DNA النواة إلى مناطق نشاطه في السايتوبلازم.

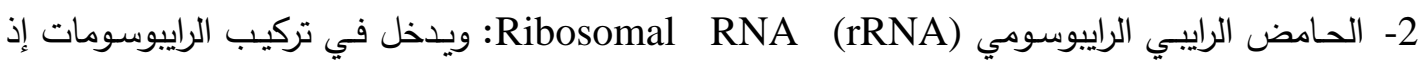
يشترك مع البروتين في بنائها.

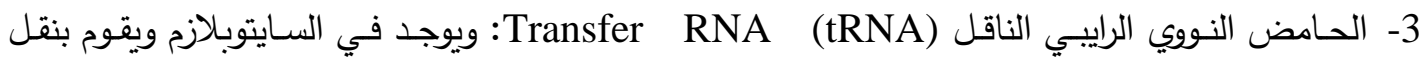
الأحماض الأمينية إلى الراييوسومات.

\section{4. توزيع بولتزمان: Boltzmann Distribution}

تعرف دالة كثافة الاحتمال لتوزيع بولتزمان بالثكل الآتي: [3]

$$
P(x ; \theta)=\left\{\begin{array}{cccc}
\exp \frac{-\Delta G(x ; \theta)}{k T} & & \text { if } & \Delta G(x ; \theta)>0 \\
1 & \text { if } & \Delta G(x ; \theta) \leq 0
\end{array}\right.
$$


إذ أن: $\Delta G$ : التغير في الطاقة، وتعرف بالصيغة الآتية:

$$
\Delta G=\sum_{x} \frac{1}{y !} \sum_{i=0}^{y-1}(-1)^{i} \frac{y !}{i !(y-i) !}(y-i)^{x} \theta
$$

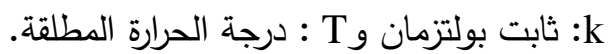
5. تقدير معلمة توزيع بولتزمان باستخدام الخوارزمية الجينية:

\section{The Estimate Parameter of the Boltzmann Distribution Using Genetic Algorithm}

سوف يتم في هذا البحث اقتراح خوارزميتين لتقدير معلمة توزيع بولتزمان باستخدام الخوارزمية الجينية

التي بدورها سوف تقوم بالمهام التالية: أ) تعظيم دالة الترجيح لتوزيع بولتزمان.

ب) تقليل قيد الجيل لتوزيع بولتزمان.

5.1. تعظيم دالة الترجيح لتوزيع بولتزمان باستخدام الخوارزمية الجينية: Maximizing the Likelihood Function of Boltzmann Distribution by Using Genetic Algorithm

سوف يتم إيجاد تقدير المعلمة $\theta$ لتوزيع بولتزمان الثرطي باستخدام طريقة الترجيح الأعظم، وهنا سنعرف سلسلة الـ (RNA ) بـ (x)، ونعرف الاحتمالية لتركيب سلسلة الـ (RNA) بـ (y )، ونستخدم النموذج الخطياللوغاريتمي الشرطي لتوزيع بولتزمان (Boltzmann Distribution) ثم نقوم بتعظيم دالة الترجيح لتوزيع بولتزمان

فيما يأتي الاشتقاق الكامل لتقدير الترجيح الأعظم (M.L.E. ) لتوزيع بولتزمـان الثرطي، إذ أن دالـة

كثافة الأحتمال لتوزيع بولتزمان الشرطي

$$
\begin{aligned}
& p(y / x, \theta)=\frac{1}{z(x, \theta)} \exp \left(\frac{-1}{k T} \Delta G(x, y, \theta)\right. \\
& \Delta G=\sum_{x} \frac{1}{y !} \sum_{i=0}^{y-1}(-1)^{i} \frac{y !}{i !(y-i) !}(y-i)^{x} \theta \\
& z(x, \theta)=\sum_{y} \exp \left(\frac{-1}{k T} \Delta G(x, y, \theta)\right) \\
& p(y / x, \theta)=\frac{\exp \left(\frac{-1}{k T} \sum_{x} \frac{1}{y !} \sum_{i=0}^{y-1}(-1)^{i} \frac{y !}{i !(y-i) !}(y-i)^{x} \theta\right)}{\sum_{y} \exp \left(\frac{-1}{k T} \sum_{x} \frac{1}{y !} \sum_{i=0}^{y-1}(-1)^{i} \frac{y !}{i !(y-i) !}(y-i)^{x} \theta\right)} \\
& \text { ولإيجاد مقدر الترجيح الأعظم لتوزيع بولتزمان الثرطي نتبع الخطوات الآتية: }
\end{aligned}
$$

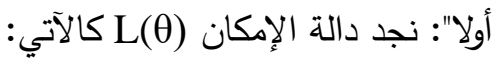




$$
L(\theta)=\prod_{r=1}^{n} p(y / x, \theta)
$$

$$
\prod_{r=1}^{n}=\sum_{m=1}^{16} \sum_{m=1}^{16}
$$

$$
\begin{gathered}
=\prod_{r=1}^{n} \frac{\exp \left(\frac{-1}{k T} \sum_{x} \frac{1}{y !} \sum_{i=0}^{y-1}(-1)^{i} \frac{y !}{i !(y-i) !}(y-i)^{x} \theta\right)}{\exp \left(\frac{-1}{k T} \sum_{x} \frac{1}{y !} \sum_{i=0}^{y-1}(-1)^{i} \frac{y !}{i !(y-i) !}(y-i)^{x} \theta\right)} \ldots(6) \\
=\left(\frac{\exp \sum_{r=1}^{n}\left(\frac{-1}{k T} \sum_{x} \frac{1}{y !} \sum_{i=0}^{y-1}(-1)^{i} \frac{y !}{i !(y-i) !}(y-i)^{x} \theta\right)}{\prod_{r=1}^{n} \sum_{y} \exp \left(\frac{-1}{k T} \sum_{x} \frac{1}{y !} \sum_{i=0}^{y-1}(-1)^{i} \frac{y !}{i !(y-i) !}(y-i)^{x} \theta\right)}\right)
\end{gathered}
$$

ثانيا": نأخذ اللوغاريتم لدالة L(O) لتصبح المعادلة كالآتي:

$$
\ln L(\theta)=\log \left(\frac{\exp \sum_{r=1}^{n}\left(\frac{-1}{k T} \sum_{x} \frac{1}{y !} \sum_{i=0}^{y-1}(-1)^{i} \frac{y !}{i !(y-i) !}(y-i)^{x} \theta\right)}{\prod_{r=1}^{n} \sum_{y} \exp \left(\frac{-1}{k T} \sum_{x} \frac{1}{y !} \sum_{i=0}^{y-1}(-1)^{i} \frac{y !}{i !(y-i) !}(y-i)^{x} \theta\right)}\right)
$$

وبتبسيط المعادلة أعلاه نحصل على ما يلي:

$\log L(\theta)=-\sum_{x=1}^{4} \sum_{y=1}^{4}\left(\ln \sum_{m=1}^{16} \sum_{m=1}^{16} \exp \left(\frac{-1}{k T} \sum_{x} \frac{1}{y(m) !} \sum_{i=0}^{y-1}(-1)^{i} \frac{y(m) !}{i !(y(m)-i) !}(y(m)-i)^{x(m)} \theta\right)-\right.$

$\left.\sum_{m=1}^{16} \sum_{m=1}^{16}\left(\frac{-1}{k T} \sum_{x} \frac{1}{y(m) !} \sum_{i=0}^{y-1}(-1)^{i} \frac{y(m) !}{i !(y(m)-i) !}(y(m)-i)^{x(m)} \theta\right)\right)$

إن دالة كثافة الأحتمال لتوزيع بولتزمان الثرطي

$$
p(y / x, \theta)=\frac{1}{z(x, \theta)} \exp \left(\frac{-1}{k T} \Delta G(x, y, \theta)\right.
$$

\section{Proposed Genetic Algorithm (1):(1) 5.3}

إيجاد قيمة المقدر الذي يعظم دالة الترجيح لتوزيع بولتزمان Finding the Value of the Estimator which Maximizes the Likelihood Function for

\section{Boltzmann Distribution}

تم استخدام دالـة الترجيح الأعظم لتوزيـع بولتزمـان في الخطوات المقترحـة للخوارزميـة الجينيـة، والخطوات

$$
\text { موضحة في أدناه : }
$$

الخطوة الأولى:- البيانات الأولية (Initial Data): وهي قراءة لمجموعة من المتغيرات التي استخدت في

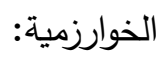

• • العداد m: وهو يمثل الطول لكل من سلسلتي X و y 
يم(m) يمثل متجـه لأحد النيوكليوتيدات الداخلـة في تركيب الـ RNA ويمثل القواعد النتروجينية:

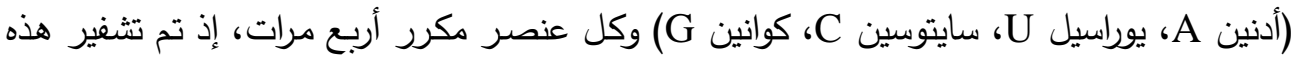

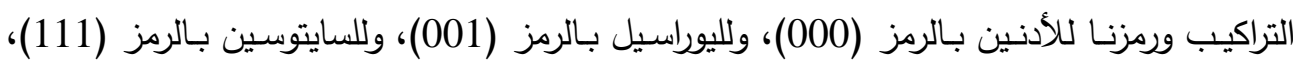
والكوانين بالرمز (010).

يمثل متجـه التراكيب الناتجـة مـن تـداخل هذه القواعد النتروجينيـة وللتكرارات أعـلاه (فقط

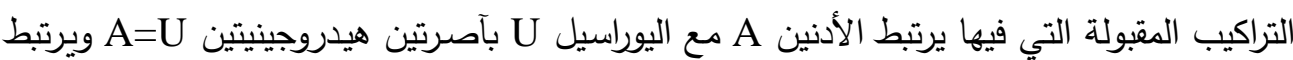

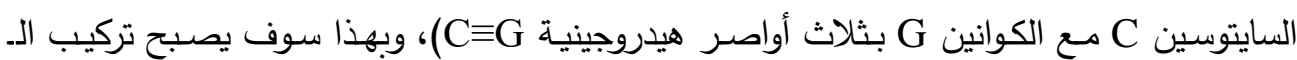

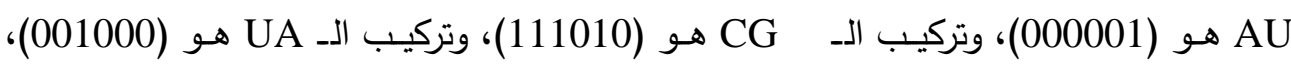
وتركيب ال GC هو (010111).

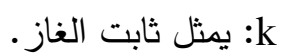

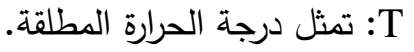
الخطوة الثانية:- إنشـاء الجيل الابتدائي (Initial Generation): الجيل الابتدائي في هذه الخوارزمية يتكون من كروموسومين احدهما يمثل القواعد النتروجينية والآخر يمثل تراكيب هذه القواعد النتروجينية ، وقد تم وضع القيم الحقيقية في جينات الكروموسومين أي أن التشفير كان تثفيرا حقيقيا للكروموسومات. الخطوة الثالثة:- قيمة الجودة ( Fitness Value): إن قيمة الجودة في هذه الخوارزمية تمثل مُقدر الترجيح الأعظم لتوزيع بولتزمان، إذ تم استخدام اللوغاريتم لأنه الأساس في استخراج المقدرات التي تجعل المقدر أعظم

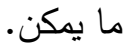

الخطوة الرابعة:- تم استخدام الانتقاء من النوعين الآتيين (Uniform Roulette، الخطوة الخامسة:- تم استخدام التعابر من الأنواع الآتية Single Point) (Scattered ،Intermediate، Heuristic الخطوة السادسة:- تم استخدام الطفرة من الأنواع الآتية (Gaussian،Uniform). وتم التحديد المسبق لعدد الأجيال في توقف الخوارزمية الجينية. والثكل الآتي يمثل المخطط الانسيابي للخوارزمية الجينية المقترحة: 


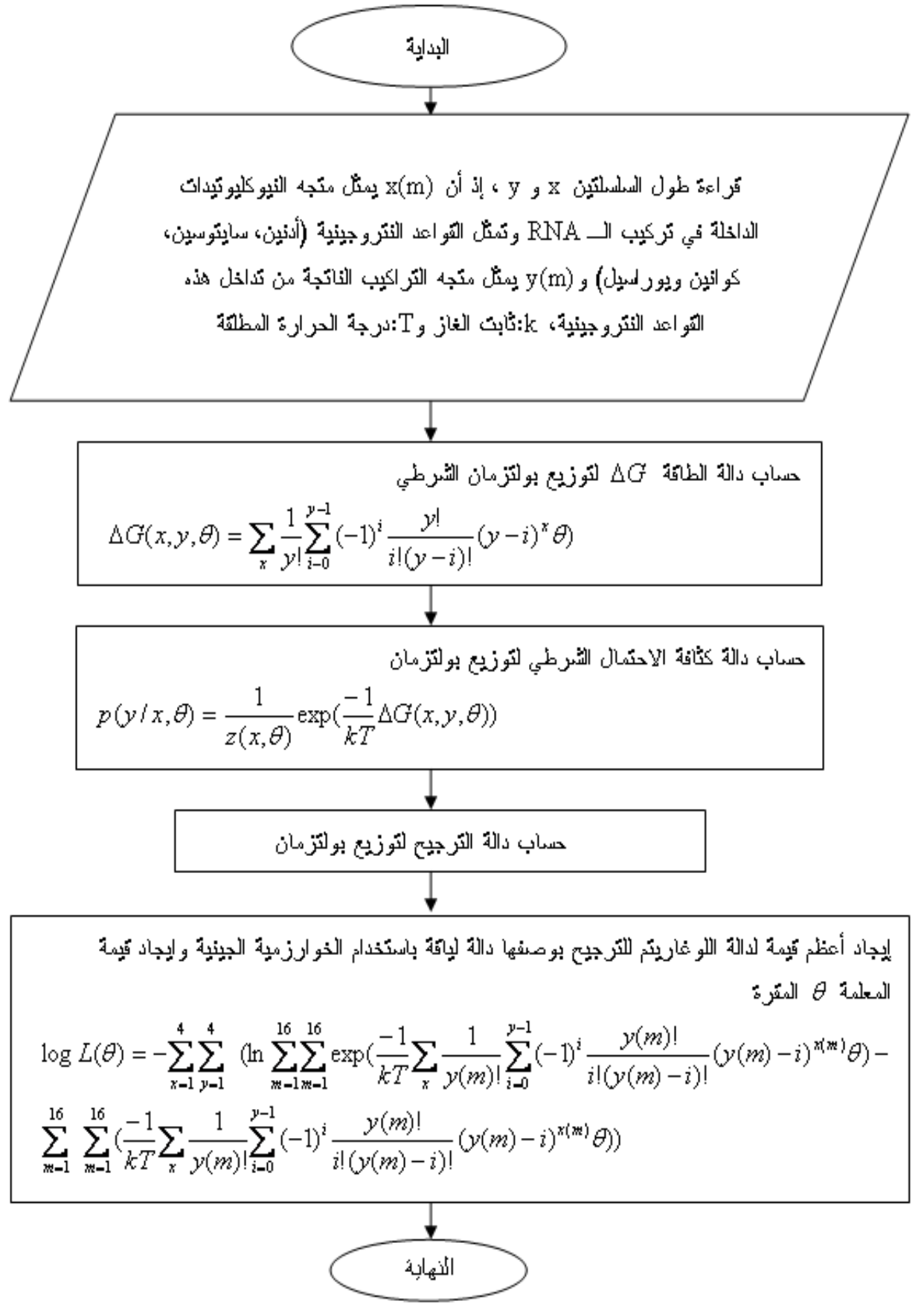

الثكل (3) يمثل المخطط الانسيابي لتقدير قيمة المعلمة الأني يعظم دالة الترجيح لتوزيع بولتزمان

تم تصميم برنامج بلغة (MATLAB) ثم تم ايجاد قيمة دالة الجودة (أعظم قيمة لدالة الترجيح) ومن خلاله تم تحديد المعلمة التي ستحقق القيمة العظمى للدالة وتم الاعتماد على التحديد المسبق لعدد الأجيال لبيان مدى التقرب من الحل الأمثل وقمنا باختيار عدة أنواع لكل من (الانتقاء ، التداخل الابدالي والطفرة) وكذلك عدد مرات توليد الأجيال وملاحظة النتائج بعد كل بيل إذ كانت أفضل النتائج لدى توليد الأجيال 
مابين 25 إلى 1500 جيل ولقد حققت هذه الخوارزمية أعظم قيمة لمقدر الترجيح لتوزيع بولتزمان عند القيمة (0.1457) والتي ظهرت عند الجيل (1387) (إذ كان:

1. الانتقاء من نوع (Roulette).

2. التداخل الابدالي من نوع (Intermediate).

3. الطفرة من نوع (Uniform).

كما موضح في الجدول (1) مونع

جدول (1) يمثل تقدير المعلمة $\theta$ بأسلوب تعظيم دالة الترجيح لتوزيع بولتزمان

\begin{tabular}{|c|c|c|c|c|c|c|}
\hline & الأجبالة & نوع الانتفَاء & 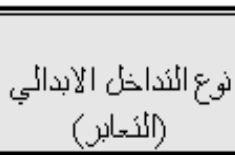 & نو ع الطُّزة & قولبمة $\theta$ & 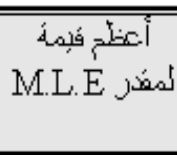 \\
\hline 1. & 100 & Roulette & Intermediate & Uniform & -21.0133 & -110.018 \\
\hline 2. & 77 & Roulette & Intermediate & Uniform & -17.1375 & -102.0289 \\
\hline 3. & 80 & Uniform & Intermediate & Gaussian & -8.0851 & -95.5772 \\
\hline 4. & 29 & Uniform & Scattered & Gaussian & 4.1539 & -94.7458 \\
\hline 5. & 100 & Uniform & Scattered & Gaussian & -12.9348 & -97.6835 \\
\hline 6. & 25 & Uniform & Single Point & Uniform & 0.0229 & -94.1751 \\
\hline 7. & 52 & Uniform & Intermediate & Uniform & 0.044 & -94.1727 \\
\hline 8. & 100 & Roulette & Scattered & Gaussian & -23.5084 & -117.5638 \\
\hline 9. & 73 & Roulette & Intermediate & Uniform & -18.9013 & -105.1023 \\
\hline 10 . & 100 & Roulette & Single Point & Uniform & 0.0084 & -94.1767 \\
\hline 11. & 171 & Uniform & Scattered & Gaussian & -7.7767 & -95.4946 \\
\hline 12. & 100 & Uniform & Single Point & Gaussian & -13.0772 & -97.7796 \\
\hline 13. & 100 & Uniform & Scattered & Gaussian & -15.6705 & -100.1023 \\
\hline 14. & 100 & Roulette & Heuristic & Uniform & -29.8607 & -142.7702 \\
\hline 15. & 100 & $\begin{array}{l}\text { Stochastic } \\
\text { Uniform }\end{array}$ & Scattered & Gaussian & -20.5965 & -108.9377 \\
\hline 16. & 84 & Roulette & Intermediate & Uniform & -18.2274 & -103.8222 \\
\hline 17. & 59 & Uniform & Heuristic & Gaussian & -17.3304 & -102.3224 \\
\hline 18. & 99 & Roulette & Intermediate & Uniform & -19.8354 & -107.105 \\
\hline 19. & 35 & Uniform & Scattered & Gaussian & -5.3125 & -94.9497 \\
\hline 20. & 49 & Uniform & Single Point & Uniform & 0.1016 & -94.1663 \\
\hline 21. & 136 & Roulette & Scattered & Uniform & 0.0815 & -94.1685 \\
\hline 22. & 234 & Uniform & Scattered & Gaussian & -1.8355 & -94.4026 \\
\hline 23. & 567 & Roulette & Intermediate & Uniform & 0.0060 & -94.1770 \\
\hline 24. & 784 & Roulette & Single Point & Gaussian & 0.0326 & -94.1740 \\
\hline 25 . & 923 & Uniform & Scattered & Uniform & 0.0435 & -94.1728 \\
\hline 26. & 419 & Roulette & Single Point & Gaussian & 45.8077 & -2164093 \\
\hline 27. & 1097 & Roulette & Heuristic & Uniform & -2.1757 & -94.4485 \\
\hline 28. & 1265 & Uniform & Scattered & Gaussian & -5.7313 & -95.0304 \\
\hline 29. & 1387 & Roulette & Intermediate & Uniform & 0.1457 & -94.1614 \\
\hline 30 . & 1500 & Uniform & Single Point & Gaussian & 4.2841 & -94.7614 \\
\hline
\end{tabular}


Minimizing تقليل قيد الجيل لتوزيـع بولتزمان باستخدام الخوارزمية الجينية: 5.4 Constraint Generation (CG) of Boltzmann Distribution Using Genetic Algorithim

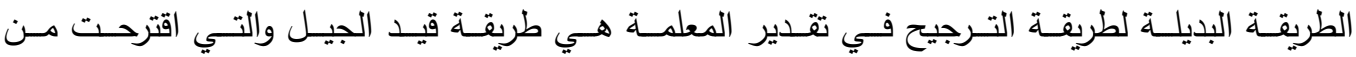

قبل Andronescu وآخرون في عام (2006) والطريقة تتلخص بتقدير قيمة $\theta$ لتوزيع بولتزمان بحل نظام القيود الموضح في المعادلة الآتية:[10]

$$
\mathrm{G}\left(\mathrm{x}, \mathrm{y}_{\mathrm{x}}, \theta\right) \leq \Delta \mathrm{G}(\mathrm{x}, \mathrm{y}, \theta)
$$

$$
\begin{aligned}
& \mathrm{Y}_{\mathrm{x}} \backslash\left\{\mathrm{y}_{\mathrm{x}}\right\} \in \mathrm{y}_{\mathrm{g}} \mathrm{S} \in\left(\mathrm{x}, \mathrm{y}_{\mathrm{x}}\right)
\end{aligned}
$$

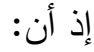

$$
\begin{aligned}
& \text { S : تمثل مجموعة بيانات تتكون من مجموعة أزواج (x,yx ) . } \\
& \text { تمثل مجموعة كل التراكيب الثانوية للمتسلسلة :xx }
\end{aligned}
$$

هذه القيود تضمن أن لكل سلسلة x جيع تراكيبها الثانوية y غير المثلى والتي يرمز لها بـ (yx) لها

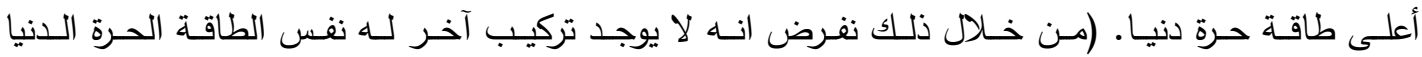
(MFE) (Minimum Free Energy)

قد يحدث أن هذا النظام من القيود يكون غير قابل للتطبيق، أي انه لا يوجد حل للمعلمة (ق) الموجودة

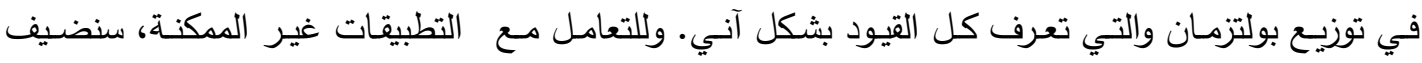

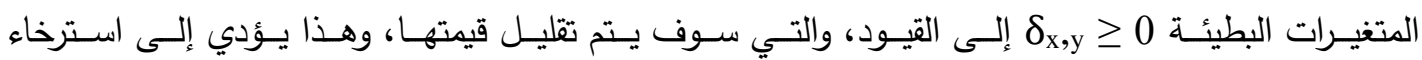
(أي تحسين قيمتها) كما موضح في المعادلة الآتية: (Relaxation)

$$
\Delta \mathrm{G}\left(\mathrm{x}, \mathrm{y}_{\mathrm{x}}, \theta\right) \leq \Delta \mathrm{G}(\mathrm{x}, \mathrm{y}, \theta)+\delta_{\mathrm{x}, \mathrm{y}}
$$

وعلى اعتبار أن دالة الطاقة هG معرفة سابقاً في المعادلة (3)، ويمكن تحويل المتراجحة أعلاه إلى

$$
\begin{aligned}
& \Delta \mathrm{G}\left(\mathrm{x}, \mathrm{y}_{\mathrm{x}}, \theta\right)-\Delta \mathrm{G}(\mathrm{x}, \mathrm{y}, \theta)=\delta_{\mathrm{x}, \mathrm{y}} \\
& \text { وبالتالي يجب أن تتحقق دالة قيد الجيل والممثلة بالمعادلة الآتية: } \\
& \text { minimize }\|\delta\|^{2} \\
& \delta \geq 0 \\
& \text { معادلة معرفة كالآتي: } \\
& \text { تقليل دالة الهدف مع تحقق قيدها. }
\end{aligned}
$$

Proposed Genetic Algorithm (2):(2) 5.5

$$
\text { إيجاد قيمة المقدر الذي يقلل قيد الجيل لتوزيع بولتزمان }
$$

Finding the Value of the Estimator which Minimizes the Constraint Generation for Boltzmann Distribution

$$
\text { موضحة في أدناه: }
$$




\section{الخطوة الأولى والخطوة الثانية كما في مثيلتيهما في الخوارزمية السابقة.}

الخطوة الثالثة:- قيمـة الجودة ( Fitness Value): إن قيمة الجودة في هذه الخوارزمية ممثلة بالمعادلة هG(x,y x,

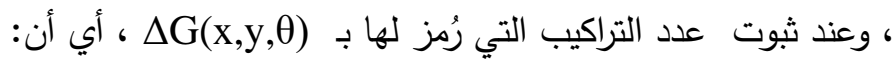

$$
\mathrm{z}=\Delta \mathrm{G}(\mathrm{x}, \mathrm{y} \text { x }, \theta)-\Delta \mathrm{G}(\mathrm{x}, \mathrm{y}, \theta)
$$

وفي هذه الحالة يجب تحقق الثرط التالي وهو أن z أكبر أو تساوي الصفر ، عندها سوف يتم حساب معيار

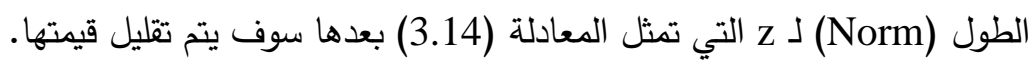

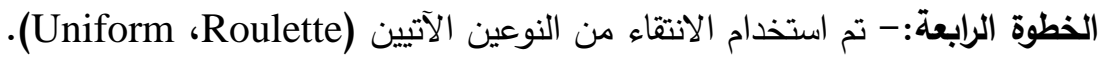

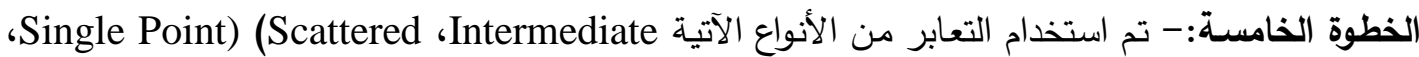
Heuristic ،Two Point الخطوة السادسة:- تم استخدام الطفرات الآتية (Gaussian،Uniform،). وتم التحديد المسبق لعدد الأجيال في توقف الخوارزمية الجينية. والثكل التالي يمثل المخطط الانسيابي للخوارزمية الجينية المقترحة: 


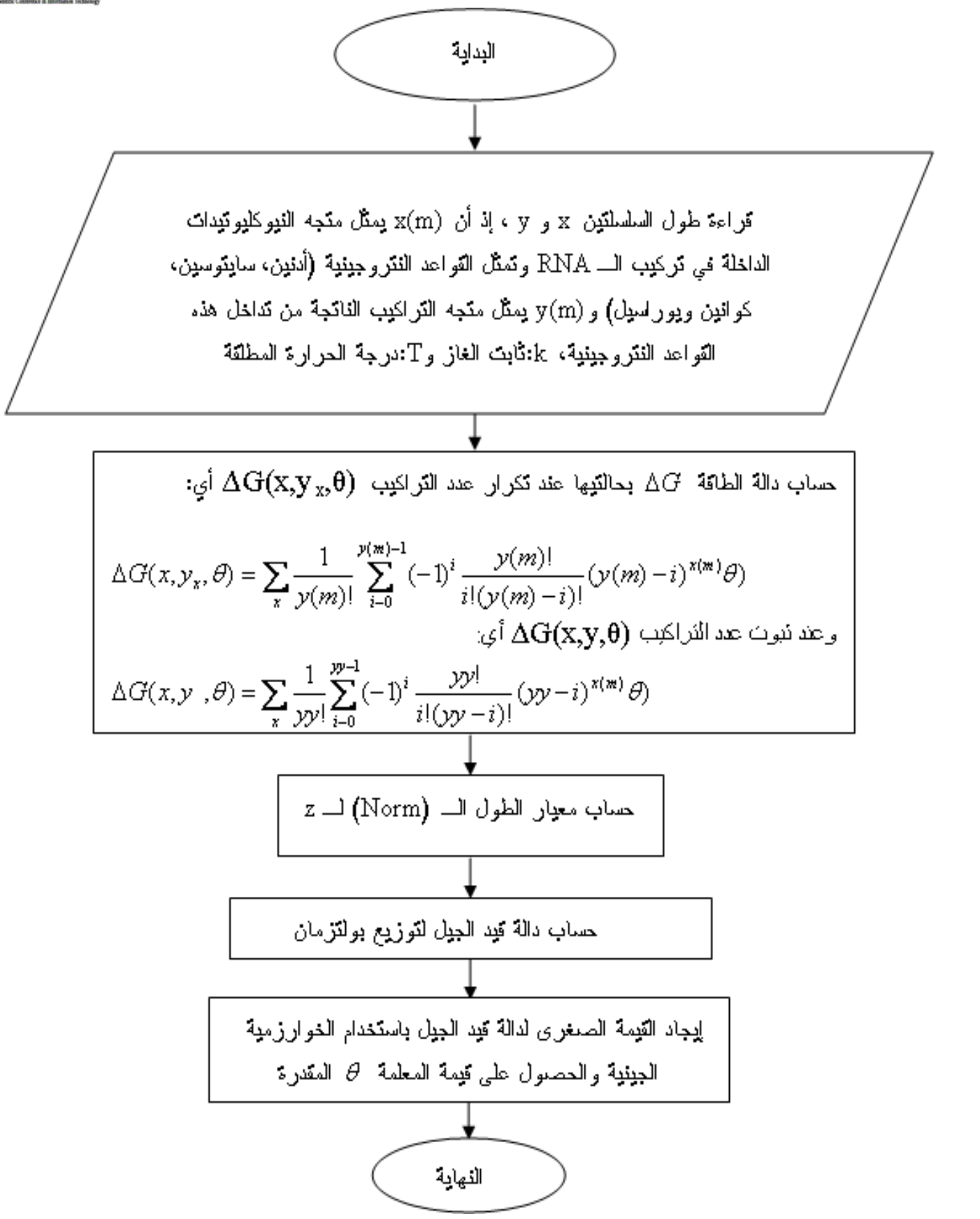

0 الثكل (4) يمثل المخطط الانسيابي لإيجاد قيمة المعلمة الأني يقلل دالة قيد الجيل لتوزيع بولتزمان

بعد أن تم ايجاد قيمة الجودة (أقل قيمة لدالة قيد الجيل)، وذلك بتصميم برنامج بلغة MATLAB ومن خلاله تم تحديد المعلمة التي ستحقق القيمة الدنيا للدالة وتم اختيار عدة أنواع لكل من (الانتقاء، التداخل الابدالي والطفرة) وكذلك عدد مرات توليد الأجيال وملاحظة النتائج بعد كل جيل إذ كانت أفضل النتائج لاى توليد الأجيال مابين 25 إلى 1500 جيل وتم الاعتماد على التحديد المسبق لعدد الأجيال لبيان مدى التقرب من الحل الأمثل ولقد حققت هذه الخوارزمية أقل قيمة لدالة قيد الجيل لتوزيع بولتزمان عند القيمة (107* (95.1039101) وقيمة $\theta$ هي (4.4066-) والتي ظهرت عند الجيل (80) إذ كان: 
1. الانتقاء من نوع (Uniform).

2. التداخل الابدالي من نوع (Intermediate).

3. الطفرة من نوع (Gaussian).

كما موضح في الجدول (2).

جدول (2) يمثل تقدير المعلمة $\theta$ بأسلوب تقليل قيد الجيل CG لتوزيع بولتزمان

\begin{tabular}{|c|c|c|c|c|c|c|}
\hline & الأجيالَ عدد & نوع الانتثاء & نوع الثتأخل الآبدالكي & نوع الطُنترة & قَيدة $\theta$ & 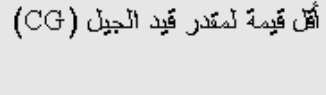 \\
\hline 1. & 64 & Roulette & Two Point & Uniform & 0.9267 & $-0.18502 * 10^{1}$ \\
\hline 2. & 100 & $\begin{array}{c}\text { Stochastic } \\
\text { Uniform }\end{array}$ & Scattered & Gaussian & -21.6482 & $-0.22952 * 10^{1}$ \\
\hline 3. & 49 & Uniform & Single Point & Uniform & 0.9944 & $-0.21303 * 10^{1}$ \\
\hline 4. & 29 & Uniform & Scattered & Gaussian & -4.6912 & $-0.10778 * 10^{1}$ \\
\hline 5. & 99 & Roulette & Intermediate & Uniform & 0.9895 & $-0.21096 * 10^{1}$ \\
\hline 6. & 100 & Roulette & Intermediate & Uniform & 0.9776 & $-0.20589 * 10^{1}$ \\
\hline 7. & 100 & Uniform & Scattered & Gaussian & -10.9584 & $-0.58814 * 10^{1}$ \\
\hline 8. & 123 & Uniform & Scattered & Gaussian & -3.4385 & $-0.579081265 * 10^{7}$ \\
\hline 9. & 80 & Uniform & Intermediate & Gaussian & -4.4066 & $-0.951039101 * 10^{7}$ \\
\hline 10. & 100 & Uniform & Single Point & Gaussian & -12.4559 & $-0.75987 * 10^{1}$ \\
\hline 11. & 52 & Uniform & Intermediate & Uniform & 0.9609 & $-0.19892 * 10^{1}$ \\
\hline 12. & 73 & Roulette & Intermediate & Uniform & 0.8569 & $-0.15820 * 10^{1}$ \\
\hline 13. & 25 & Uniform & Single Point & Uniform & 0.9916 & $-0.21184 * 10^{1}$ \\
\hline 14. & 100 & Uniform & Scattered & Gaussian & -6.5909 & $-0.21276 * 10^{1}$ \\
\hline 15 & 84 & Roulette & Intermediate & Uniform & 0.8834 & $-0.16815 * 10^{1}$ \\
\hline 16. & 59 & Uniform & Heuristic & Gaussian & -26.4713 & $-0.34319 * 10^{1}$ \\
\hline 17. & 350 & Uniform & Scattered & Gaussian & -1.7094 & $-0.143105685 * 10^{7}$ \\
\hline 18 & 100 & Roulette & Heuristic & Uniform & 1.3858 & $-0.41378 * 10^{1}$ \\
\hline 19. & 77 & Roulette & Intermediate & Uniform & 0.9625 & $-0.19961 * 10^{1}$ \\
\hline 20. & 100 & Roulette & Scattered & Gaussian & -21.5456 & $-0.22736 * 10^{1}$ \\
\hline 21. & 178 & Uniform & Heuristic & Gaussian & -50.4029 & $-0.12442 * 10^{1}$ \\
\hline 22. & 232 & Roulette & Intermediate & Gaussian & -7.8907 & $-0.304943 * 10^{1}$ \\
\hline 23. & 489 & Uniform & Scattered & Uniform & 0.9889 & $-0.21066 * 10^{1}$ \\
\hline 24. & 678 & Uniform & Intermediate & Gaussian & -0.5562 & $-0.15151389 * 10^{6}$ \\
\hline 25 . & 81 & Uniform & Intermediate & Gaussian & -8.5679 & $-0.35953 * 10^{1}$ \\
\hline 26. & 1010 & Uniform & Scattered & Gaussian & -1.8179 & $-0.16184835810^{7}$ \\
\hline 27. & 1215 & Roulette & Scattered & Uniform & -5.2872 & $-0.13691 * 10^{1}$ \\
\hline 28. & 1365 & Uniform & Intermediate & Gaussian & -1.4349 & $-0.100842706 * 10^{7}$ \\
\hline
\end{tabular}




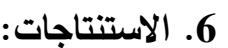

إيجاد قيمـة المقدر الذي يعظم دالة الترجيح لتوزيع بولتزمان باستخدام الخوارزميـة الجينيـة المقترحة الأولى استُتج ما يأتي:

أعظم قيمة لمقدر الترجيح لتوزيع بولتزمان ظهرت:

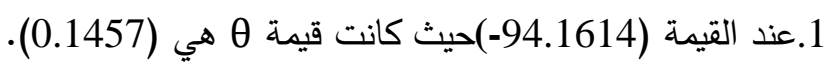

2. (1387) (138).

3. الانتقاء من نوع (Roulette).

4.كذلك التداخل الابدالي من نوع (Intermediate).

5.الطفرة من نوع (Uniform).

إيجاد قيمة المقدر الذي يقلل قيد الجيل لتوزيع بولتزمان باستخدام الخوارزمية الجينية المقترحة الثانية استُتج ما

أقل قيمة لقيد الجيل لتوزيع بولتزمان ظهرت:

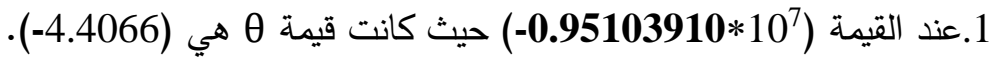

2.

3.الانتقاء من نوع (Uniform).

4.التداخل الابدالي من نوع (Intermediate).

5.الطفرة من نوع (Gaussian).

استنتجنا ممـا سبق بـأن الخوارزميـة الجينيـة كانت الأسـلوب الأفضـل لتحقيق أعظم قيمـة لدالـة الترجيح

والحصول على أمثل قيمة للمعلمة $\theta$ وكذلك حقتت أدنى قيمة لدالة قيد الجيل والحصول أيضـاً على أمثل قيمة

لمعلمة 


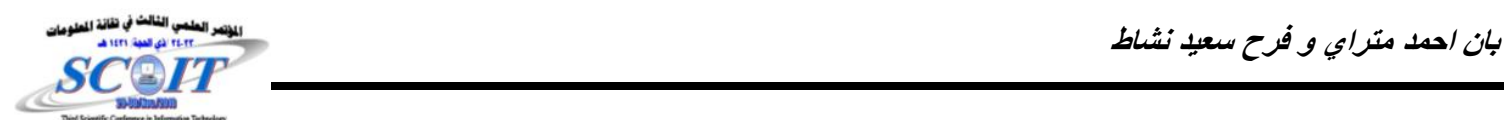

$$
\begin{aligned}
& \text { المصاد } \\
& \text { [1]. ثابت، همسـة معن. (2005)، "بعض تطبيقـات الخوارزميـة الجينيـة في حل مسـائل الأمثليـة"، رسالة } \\
& \text { ماجستير ، جامعة الوصل، كلية علوم الحاسبات والرياضيات، قسم الإحصاء. } \\
& \text { ذنـون، باسـل يـونس (1988)، "الإحتماليـة والمتغيـرات العثـوائية"، مديريـة دار الكتب والنشـر ، جامعـة }
\end{aligned}
$$

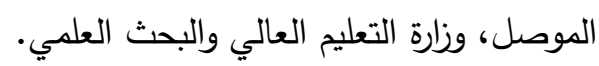

[3]. Andronescu, M.; Condon, A.; Hoos, H. H.; Mathews, D. H. and Murphy, K., (2006), " Efficient Parameter Estimation for RNA Secondary Structure Prediction", (C) Oxford University Press.

[4]. Chan, C. Y. and Ding, Y., (2008), " Boltzmann Ensemble Features of RNA Secondary Structures: a Comparative Analysis of Biological RNA Sequences and Random Shuffles", C. Y. Chan · Y. Ding Wadsworth Center, New York State Department of Health, Center for Medical Science, 150 New Scotland Avenue, Albany, NY 12208, USA.

[5]. Devore, J. R., (2000), "Probability and Statistics", Brooks, Cole.

[6]. Ding, Y. and Lawrence, C. E., (2003), "A statistical Sampling Algorithm for RNA Secondary Structure Prediction. Nucleic Acids Research", 31: pp. 7280-7301.

[7]. Ding, Y., (2006), "Statistical and Bayesian Approaches to RNA Secondary Structure Prediction", Published by Cold Spring Harbor Laboratory Press, 12: pp. 323-331.

[8]. Ding, Y.; Chan, C. Y. and Lawrence, C. E., (2006), " Clustering of RNA Secondary Structures with Application to Messenger RNAs", Elsevier Ltd, 0022-2836.

[9]. Irwin, H. H., (1967), "Basic Principle of Molecular Genetice", Nelson Ltd. London.

[10]. Pond, K. S. L.; Mannino, F. V. ; Gravenor, M. B.; Muse, S. V. and Frost, S. D. W., (2006), "Evolutionary Model Selection with a Genetic Algorithm: A Case Study Using Stem RNA", Oxford University Press on Behalf of the Society for Molecular Biology and sEvolution. Moscow.

[11]. William, T. T. and Richared, J. W., (1968), "General Biology", $2^{\text {nd }}$ Edition, Van Nostrand Reinhold Company.

[12]. "Genetic Algorithms", the web site at http://www.cs.felk.cvut.cz/ xobitko/ga/main.html Obitko M. (2009)

[13]. Marczyk, A., (2004), "Genetic Algorithm and Evolutionary Computation", April, Vol. 23.

http://www.talkor:gins.org/Faqs/genalg/genalg.html/\#introduction. 